\title{
ACTIVIDAD in vitro DE PROGESTERONA EN LOS SEGMEN- TOS CRANEAL, MEDIO Y CAUDAL DE CUERNOS UTERINOS GRÁVIDOS DE COBAYO (Cavia porcellus)
}

\author{
Ericka Otárola G. ${ }^{1}$, Milder Ayón S. ${ }^{2}$ y Sergio Cueva M. ${ }^{2}$
}

\section{Abtrat}

The action of hydroxyprogesterone capronate on the muscular activity of the cranial, medial and caudal segments of the right and left uterine horns of 15 primiparous guinea pigs (Cavia porcellus) at 10-12 days gestation was examined. Additive doses of progesterone were given until a maximum response of $23.813 \times 10^{-7} \mathrm{mM}$ was achieved. The percentage of subsequent muscular relaxation of both horns was measured in relation to maximum muscular tension (determined by depolarization of the membrane with $\mathrm{KCl}$ ). The greatest degree of muscular relaxation occurred in the cranial $(104.23 \pm \pm 2.89$ and $127.06 \pm \pm 3.09$ for the left and right horns, respectively) and caudal (110.41 \pm \pm 3.18 and $70.14 \pm \pm 2.18$ for the left and right horns, respectively) segments of the uterus. Although lesser values $(\mathrm{P}<0.05)$ were found for the medial segment $(54.45 \pm \pm 2.24$ and $50.82 \pm \pm 1.72$ for the left and right horns, respectively), the greatest proportion of embryos were implanted here $(35.42 \%$ and $25.00 \%$ in the left and right horns, respectively), suggesting that the progesterone has not yet established its inhibitory role over muscular activity. Key words: progesterone, uterine horn, miometrium, smooth muscular contraction, $\mathrm{Ca}$ via porcellus, guinea pig.

\section{Ravomen}

Se estudió la actividad del capronato de hidroxiprogesterona sobre la actividad muscular de los segmentos craneal, medio y caudal de los cuernos uterinos izquierdo y derecho de 15 cobayas (Cavia porcellus) gestantes, de primer parto, entre 10 y 12 días de gestación a fin de entender su probable relación con la muerte embrionaria. La hormona fue aprobada en dosis aditivas, obteniendo la máxima respuesta con $23.813 \times 10^{-7} \mathrm{mM}$ de progesterona. Estas ejercieron relajación muscular de ambos cuernos expresada en porcentaje de máxima tensión muscular obtenida previamente por despolarización con CIK. Se observó menor relajación $(\mathrm{P}<0.05)$ en los segmentos medios $(54.45 \pm 2.24$ y $50.82 \pm 1.72$ en los cuernos izquierdo y derecho, respectivamente), que en los segmentos craneales ( $104.23 \pm 2.89$ y $127.06 \pm 3.09$ en los cuernos izquierdo y derecho, respectivamente) y caudales ( $110.41 \pm 3.18$ y $70.14 \pm 2.18$ en los cuernos izquierdo y derecho, respectivamente). A pesar de encontrar la menor relajación de los segmentos medios, en ellos se localizó el mayor número de embriones (35.42\% en el cuerno izquierdo y $25.00 \%$ en el cuerno derecho). Estos hechos, en este período de gestación de la cobaya, podrían estar relacionados con grandes cambios hormonales, donde probablemente la progesterona aún no esté inhibiendo plenamente la actividad muscular uterina en los segmentos medios.

Palabras clave: progesterona, cuerno uterino, miometrio, contracción muscular lisa, $\mathrm{Ca}$ via porcellus, cobayo.

1 Práctica privada

2 Laboratorio de Fisiología FMV - UNMSM. E.mail: d170006.unmsm.edu.pe 


\section{Tritortwotion}

En el primer tercio de la gestación, ocurre la migración intrauterina del embrión, que coincide con la fase de alargamiento, rápido crecimiento del blastocisto y receptividad del útero. En multíparas ocurre con mayor frecuencia degeneración de los óvulos fecundados. Es posible que durante el primer trimestre de gestación desaparezcan entre el 10 y $40 \%$ de los embriones por muerte precoz en vacas "repetidoras", ovejas, cobayas, ratonas y huronas, (Kolb, 1979; Paria et al., 1998). En Camélidos Sudamericanos se registra también una elevada pérdida embrionaria (Fernández-Baca et al., 1970) durante el primer mes de gestación.

Durante la gestación, la progesterona es necesaria para la anidación y desarrollo del huevo, permite la implantación del blastocisto y el desarrollo en el endometrio de células deciduales. Además, junto a otras hormonas brinda el medio requerido por el embrión a fin de sostener la gestación (Paria et al., 1998). La progesterona también puede inhibir el receptor de oxitocina (Whates y Lamming, 1995) y de la misma progesterona (Chetrite y Pasqualini, 1991), sus efectos en los tejidos se hacen evidentes cuando éstos han estado previamente expuestos a los estrógenos, los cuales dependiendo de la dosis, actúan incrementando el número de receptores para esta hormona. (Janne, 1981; Murray y Stone, 1989).

Con el objeto de lograr un mejor entendimiento del rol que juega la progesterona sobre el útero y su probable relación con las pérdidas embrionarias es de interés conocer su efecto sobre la actividad muscular de las diversas porciones de los cuernos uterinos. De allí la importancia de este estudio que usa como modelo al cobayo.

\section{Materiales y Mtetudos}

El experimento se llevó a cabo en el Laboratorio de Anatomía y Fisiología del De- partamento Académico de Ciencias Veterinarias Básicas de la Facultad de Medicina Veterinaria de la Universidad Nacional Mayor de San Marcos. Se utilizaron 15 hembras de Cavia porcellus de primer parto que fueron servidas en el primer celo post-parto, de tal forma que quedaron preñadas y fueron sacrificadas a los 10 - 12 d. de gestación.

Para el estudio se utilizó el método descrito por Kasuya et al. (1988). Inmediatamente después de sacrificados los animales se evaluaron las características morfométricas de los cuernos uterinos y se anotó el número y ubicación de los embriones. Topográficamente los cuernos uterinos, derecho e izquierdo fueron divididos en tres segmentos iguales, tomándose de la porción media de cada segmento una muestra de $1 \mathrm{~cm}$ de longitud. Luego, cada muestra se llevó al sistema de cultivo in vitro que contenía líquido nutricio, Krebs-Ringer de la siguiente composición en mM: $\mathrm{NaCl}$ 113.0, $\mathrm{KCl} 4.8, \mathrm{CaCl} 2$ 2.2, $\mathrm{MgCl} 21.2$, NaHCO3 25.0, KH2PO4 1.2 y Glucosa 5.5. La solución fue mantenida a un $\mathrm{pH}$ de $7.0 \mathrm{a}$ 7.4 y a una temperatura de $37^{\circ} \mathrm{C}$ y con un sistema de oxigenación.

Cada segmento uterino fue cultivado en $10 \mathrm{ml}$ de la solución Krebs-Ringer, la cual fue cambiada cada 20 minutos. En un promedio de 2 horas se logró la tensión óptima de $1 \mathrm{~g}$, indispensable para obtener la respuesta de despolarización de los tejidos con cloruro de potasio $(\mathrm{KCl})$. A partir de la tensión óptima se midieron los cambios observados como respuesta del tejido. La máxima contracción del músculo uterino obtenida por despolarización de la membrana mediante cambios en la concentración de electroquímicos debido a $50 \mathrm{mM}$ $\mathrm{KCl}$ fue considerado como el $100 \%$ de la actividad del músculo uterino (contracción máxima). Esta respuesta sirvió de referencia para obtener los porcentajes de respuesta a las diferentes dosis acumulativas de capronato de hidroxiprogesterona, que se aplico con la ayuda de una micropipeta graduable en las siguientes concentraciones: $7.954 \times 10^{-7} \mathrm{mM}$ (dosis 1$), 15.891 \mathrm{x}$ 
$10^{-7} \mathrm{mM}$ (dosis 2), $23.813 \times 10^{-7} \mathrm{mM}$ (dosis 3) y $47.485 \times 10^{-7} \mathrm{mM}$ (dosis 4 ).

Análisis estadístico: Luego de una transformación a $\log 10$, se realizó un análisis de varianza (Anova) para comparar las respuestas de los segmentos craneal, medio y caudal de ambos cuernos uterinos. Se realizó la prueba de Tukey $(\mu=0.05)$ para las interacciones. Se realizó la prueba de Chi cuadrado para comparar la ubicación de los embriones en los segmentos de los cuernos uterinos $(\mu=0.05)$.

\section{Resullados y Discusión}

$\mathrm{Al}$ analizar la respuesta in vitro de los segmentos frente a las dosis aditivas de progesterona se encontró que a mayor dosis hubo mayor respuesta. El no encontrar diferencia estadística significativa entre las dosis 3 y 4 aplicadas, pero sí con las dosis 1 y 2 ( $\mathrm{P}<0.05)$, indica a la dosis 3 como responsable de la máxima respuesta para la progesterona. (Cuadro 1).

Cuadro 1. Valores promedio de relajación muscular lisa (porcentaje de máxima tensión con $\mathrm{ClK}$ ) de cuernos uterinos de cobayas de 10 a 12 días de gestación por acción de dosis aditivas de progesterona en $\mathrm{mM}$.

\begin{tabular}{ccc}
\hline & Dosis & $\mathrm{X} \pm \mathrm{DE}$ \\
\hline 1 & $7.954 \times 10^{-7}$ & $45.29 \pm 2.68^{\mathrm{a}}$ \\
2 & $15.891 \times 10^{-7}$ & $78.70 \pm 3.09^{\mathrm{b}}$ \\
3 & $23.813 \times 10^{-7}$ & $101.62 \pm 3.28^{\mathrm{c}}$ \\
4 & $47.485 \times 10^{-7}$ & $118.85 \pm 3.47^{\mathrm{c}}$ \\
\hline
\end{tabular}

a,b,c: Letras diferentes indican diferencia estadística significativa $(\mathrm{P}<0.05)$.

La administración de $23.813 \times 10^{-7}$ $\mathrm{mM}$ de progesterona en todos los segmentos de ambos cuernos uterinos de cobayas entre los 10 a 12 días de gestación, indujo relajación muscular de diferente grado (Cuadro 2). Estos resultados concuerdan con los reportados por Goldsmith et al. (1989); Zhu et al. (1995) Stirnemann et al. (1997) y Groothuis et al. (1997) quienes describen que la progesterona produce relajación de músculo liso en útero durante la etapa temprana de gestación de mamíferos incluyendo el hombre (Juárez-Bengoa y Perusquia, 1997), por lo que la quietud uterina durante la preñez se atribuye a esta hormona (Haluska et al., 1997; Glasier y Hobkirck, 1993) que también regula el acumulo de fluidos y proteínas en el lumen ute- rino (Moffatt et al., 1987) e inhibe la sensibilidad uterina miometrial del sistema calcio-calmodulina (Fujii et al., 1993; Fomin et al., 1999).

No se encontró diferencia significativa de la actividad de progesterona entre los cuernos uterinos izquierdo y derecho ni entre los segmentos craneal y medio de ambos cuernos; pero sí hubo diferencia $(\mathrm{P}<0.05)$ entre los segmentos caudales. Al comparar la actividad de progesterona entre los segmentos en cada cuerno, la menor relajación $(\mathrm{P}<0.05)$ se produjo en los segmentos medios de ambos cuernos uterinos, segmentos en los cuales a su vez, se ubicaron la mayor cantidad de embriones (Cuadro 3). 
Cuadro 2. Valores promedio de máxima relajación muscular lisa (referido al \% de máxima tensión por $\mathrm{ClK}$ ) en los segmentos craneal, medio y caudal de cuernos uterinos de cobayas de 10 a 12 días de gestación, por efecto de $23.813 \times 10^{-7} \mathrm{mM}$ de progesterona.

\begin{tabular}{lcc}
\hline Segmento & $\begin{array}{c}\text { Cuerno Derecho } \\
\mathrm{x} \pm \mathrm{DE}\end{array}$ & $\begin{array}{c}\text { Cuerno Izquierdo } \\
\mathrm{x} \pm \mathrm{DE}\end{array}$ \\
\hline Craneal & $127.06 \pm 3.09^{\mathrm{a}}$ & $104.23 \pm 2.89^{\mathrm{a}}$ \\
Medio & $50.82 \pm 1.72^{\mathrm{b}}$ & $54.45 \pm 2.24^{\mathrm{b}}$ \\
Caudal & $70.14 \pm 2.18^{\mathrm{c}}$ & $110.41 \pm 3.18^{\mathrm{a}}$ \\
\hline
\end{tabular}

a,b,c: Letras diferentes indican diferencia estadística significativa $(\mathrm{P}<0.05)$ en líneas y columnas.

Cuadro 3. Número de embriones y su ubicación en los segmentos craneal, medio y caudal de cuernos uterinos de cobayas de 10 a 12 días de gestación.

\begin{tabular}{lcccc}
\hline \multirow{2}{*}{ Segmento } & \multicolumn{2}{c}{ Cuerno Derecho } & \multicolumn{2}{c}{ Cuerno Izquierdo } \\
\cline { 2 - 5 } Craneal & $\mathrm{n}$ & $\%$ & $\mathrm{n}$ & $\%$ \\
Medio & 4 & $8.34^{\mathrm{a}}$ & 1 & $2.08^{\mathrm{a}}$ \\
Caudal & 12 & $25.00^{\mathrm{b}}$ & 17 & $35.42^{\mathrm{b}}$ \\
Total & 7 & $14.58^{\mathrm{a}}$ & 7 & $14.58^{\mathrm{a}}$ \\
\hline
\end{tabular}

$\mathrm{n}$ : Número de embriones

a,b: Letras diferentes, significa diferencia estadística significativa $(\mathrm{P}<0.05)$

Estos resultados estarían relacionados con la ubicación, concentración y/o activación de receptores para progesterona así como la oportuna interacción de ésta con otras hormonas y sus receptores, siendo necesaria su interacción para el establecimiento del blastocisto y desarrollo embrional. (Loza, 1994; Loza, 1991). Durante la gestación temprana los receptores para la progesterona están presentes en el estroma y miometrio, dependiendo este incremento de la dosis de estradiol, (Geisert et al., 1994; Sumida et al., 1988), los efectos de la progesterona en los tejidos se hacen evidentes cuando éstos han estado previamente expuestos a los estrógenos, los cuales actúan incrementando el número de receptores para esta hormona (Janne, 1981; Murray y Stone, 1989; Geisert et al., 1994).

Los resultados del presente trabajo podrían llevar a la interrogación de por que, si es en los segmentos medios donde se observó la mayor ubicación de embriones (Cuadro 3), por que en ellos no se obtuvo la mayor relajación uterina. Redmond (1994) determinó en ratas en el primer tercio de gestación que la mayor concentración de progesterona 
y sus metabolitos se observó en los lugares de implantación que en los lugares de interimplantación es evidente que sus resultados están referidos solamente a estos lugares. Esto podría sugerir que en el presente estudio la menor relajación uterina en el segmento medio, la progesterona no tendría el rol protagónico entre los 10 y 12 de gestación.

En esta etapa, los niveles de prostaglandina $E 2$ en sangre periférica y vena uterina están altos (Dave et al., 1983; Olofsson et al., 1990) lo que podría estar influyendo en este segmento, Las prostanglandinas, especialmente $\mathrm{E} 2$ y F2 son consideradas importantes para el espaciado del blastocisto, implantación y placentación en el útero de roedores, siendo expresados los receptores para estas hormonas de manera temporal y celular específica en la periimplantación en el útero del ratón (Yang et al., 1997). Podría suceder que en condiciones fisiológicas normales, serían los segmentos medio y caudal donde mayor número de factores deben interactuar con la progesterona para producirse la implantación.

De los resultados del presente trabajo, podríamos asumir que en cobayas dentro de los diez y doce días de gestación aún no existe una total implantación del embrión y que podría estar aún en el proceso migratorio.

\section{Conclusines}

La hormona progesterona relajó el tejido miometrial uterino entre los días 10 y 12 de gestación, tanto en el cuerno derecho como en el izquierdo.

Indistintamente, los embriones se ubicaron entre el cuerno izquierdo y cuerno derecho, sin embargo, es en el segmento medio de ambos cuernos uterinos a los 10 y 12 días de gestación donde se observó el mayor porcentaje de embriones y la menor relajación muscular $(\mathrm{P}<0.05)$.

\section{Literntura Citath}

1. Chetrite G. y J. Pasqualini. 1991. Biological responses of ICI 164,384 and other antiestrogens in vaginal and uterine cells of fetal guinea pig in culture. Acta Endocrinal. 125(4):401-408.

2. Dave J., L. Richardson y R. Knazek. 1983. Prolactin-binding capacity, prostaglandin synthesis and fluidity of murine hepatic membranes are modified during pregnancy and lactation. $J$ Endocrinol. 99(1):99-106.

3. Fernández-Baca, S., W. Hamsel y $C$. Novoa. 1970. Embryonic mortality in the alpaca. Biol. of Reprod. 3:243-251.

4. Fomin V.P., B.E. Cox y R.A. Word. 1999. Effect of progesterone on intracellular $\mathrm{Ca}^{2+}$ homeostasis in human myometrial smooth muscle cells. Am. J. Physiol. 276(2 Pt 1): C379-385.

5. Fujii E., M. Oku, S. Adachi, T. Otsuki, M. Ichijo y K. Morimoto. 1993. Effects of steroid hormones on change in $\mathrm{CC} 2$ + ) following oxytocin estimulation in culture human myometrial cells and on myometrial oxytocin receptors. Nippon Sanka. Fujinka Gakkai Zasshi. 45(7):636 -642 .

6. Geisert R., T. Pratt, F.W. Bazer, J.S. Mayes y G.H. Watson. 1994. Inmunocytochemical localization and changes in endometrial progestine receptor protein during the porcine oestrous cycle and early pregnancy reprod. Fertil. Dev. 6(6):749-760.

7. Glasier M. y R. Hobkirk. 1993. Nuclear receptors for progesterone and stradiol in the Guinea pig uterine comparment during gestation. Steroid. 58 (10):468-483.

8. Goldsmith L., J. Bkurnik, A. Wojtlauk, M. Linden, M.J. Kuhar y G. Weiss 1989. The antagonist effect of oxytocin and relaxine on rat uterine segment contractility. Am. J. Obstet. Ginecol. 161: 1641-1648.

9. Groothuis $P$., R. Blair, R. Simmen, J.L. Vallet, D.M. Grierger y D.L. Davies. 1997. Uterine response to 
progesterone in prepuberal gilts. J. Reprod. Fertil. 110(2):237-243.

10. Haluska G., M. Cook y M. Noby. 1997. Inhibition and augmentation of progesterone production during pregnancy: effects on parturition in Rhesus monkey. Am. J. Obstet. Gynecol. 176(3):682-691.

11. Janne O. 1981. Progesterone action in mamalian uterus. Acta Obstet. Gynecol. Scan. Suppl. 101:11-16.

12. Juarez-Bengoa A. y M. Perusquia. 1997. Modulation of uterine contractile activity by steroid sex hormones. Ginecol. Obstet. México. 65:498-503.

13. Kasuya $\quad$ Y., $\quad$ T. Ishikawa, M. Yanagisawa, S. Kimura, K. Goto y T. Masaki. 1988. Mechanism of contraction to endothelin in isolated porcine coronary artery. Am. J. Physiol. 257(26): H-828 - H-835

14. Kolb E. 1979. Fisiología veterinaria. Vol. 2. España. Acribia. p. 782 - 787, 1636 1642.

15. Loza M. 1994. The role of embryonary steroidogenesis in early pregnancy. Ginecol. Obstet. 62(1):17-22.

16. Loza M. 1991. Hormonal requirements for blastocyst implatation. Ginecol. Obstet. 59:347-352.

17. Moffat R., F. Bazer, R. Roberts y W.W. Thatcher. 1987. Secretory function of the ovine uterus: effects of gestation and steroid replacement therapy. J. Anim. Sci. 65(5): 1400-1410.

18. Murray C. y G. Stone. 1989. Delayed response of the mouse uterus to progesterone and oestradiol. Reprod. Fertil. Dev. 1(2):107-115.

19. Olofsson J., E. Norjavaara y G. Selstam. 1990. In vivo levels of prostaglandin F2 alpha, E2 and prostacyclin in the corpus luteum of pregnant and pseudopregnant rats. Biol Reprod. 42(5-6):792-800.

20. Paria BC., H. Lim, X.N.Wang, J. Liechr, S.K. Das y S.K. Dey. 1998. Correlation of diffential effect of primary estrogen and catecholestrogen on two distinct targets mediates embryo implantation in the mouse. Endocrinology. 139(12):5235-5246.

21. Redmond A. 1994. Progesterone and progesterone metabolite concentration in implantation sities in the pregnant rat. Life Sci. 55(23):1863-1870.

22. Stirnemann B., B. Bouftila y $M$. Clabaut. 1997. Increase of myometral activity correlated with variation in 17 beta estradiol and progesterone uterine concentration in mid - term pregnant rat estrogen agonist effect of 4 hidroxitamoxifen. Acad. Sci. III. 320 (1): 19-25.

23. Sumida C., C. Gelly y J. Pasqualini. 1988. Control of progesterona receptors in fetal uterine cells in culture: effects of stradiol, progestins, antiestrogens and growth factors. Endocrinology. 122(1):3 -11 .

24. Whates D. y G. Lamming. 1995. The oxytocin receptor, luteolysis and the maintenance of pregnancy. J. Reprod. Fertil. Suppl. 49:53-67.

25. Yang Z., S. Das, J. Wang, J. Sugimoto, A. Ichikawa y S.K. Dey. 1997. Potential sites of prostaglandin actions in the periimplatation mouse uterus: differential expression andregulation of prostaglandin receptor genes.

26. Zhu L., M. Bagchi y I. Bagchi. 1995. Ferritin heavy chain is a progesteroneinducible marker in the uterus during pregnancy.Endocrinology.136(9):4106-4115 